\title{
Control Based Sensor Management
}

\section{for a Multiple Radar Monitoring Scenario}

\author{
Yong Xun and Mieczyslaw M. Kokar \\ Department of Electrical and Computer Engineering \\ Northeastern University, Boston, Massachusetts \\ yxun@ece.neu.edu and kokar@coe.neu.edu \\ Kenneth Baclawski \\ College of Computer Science \\ Northeastern University, Boston, Massachusetts \\ kenb@ccs.neu.edu
}

\begin{abstract}
In this paper we consider the problem of monitoring illuminations of multiple emitters by one electronic receiver located on a moving platform. The emitters exhibit a quasi-periodic radiation pattern, each in a different frequency band and with a different illumination period and illumination time. The goal is to tune the receiver to the appropriate frequency band at the appropriate time so as to maximize the probability of detection of each illumination of each radar (expressed as a quality of service metric). This problem can be viewed as two subproblems: (1) generating tuning requests by particular frequency bands and (2) scheduling the receiver to satisfy the requests. In our scenario the number of requests from all the bands exceeds the physical capability of one receiver (overload) and thus selection is needed. The goal is to push the limits on the overload while still maintaining a required level of quality of service metric. This can be achieved by controlling both the generation of the tuning requests and the scheduling. According to the control theory metaphor of software development, we map this problem onto a control architecture with one system-level controller and a collection of emitterlevel controllers (one per emitter). We show that our control based sensor manager has significant advantages over a scheduler without feedback in terms of both the overload and the quality of service metric. We also discuss design issues of such sensor management systems.
\end{abstract}

Keywords: multiple radar monitoring, real-time task generation, real-time scheduling, real-time resource allocation, sensor scheduling, sensor management 


\section{Introduction}

Modern avionics systems carry a number of sensors for collecting data about the environment. Normally the demands for sensing are much higher than the physical capabilities that the sensors can provide. As a consequence, the sensors must be used selectively, balancing the conflicting sensing requests from the point of view of the goal of the mission of the avionics system. The research domain that deals with scheduling sensors is called Sensor Management. According to [1], the goal of a sensor management system is to "select the right sensor to perform the right service on the right object at the right time".

In this paper we consider the problem of managing sensors that monitor sources of electronic radiation in the environment (such as radars). The sources emit radiation in different frequencies and with various illumination patterns and periodicities. The goal is to detect each illumination of each emitter. In other words, the goal is one of monitoring all the emitters and not just detecting active radars. Information about each illumination of a radar can be used by on-board electronics for countermeasures like deception and jamming.

In our scenario an omni-directional receiver is tuned to a particular frequency band to detect a radar emitting in this frequency band. Since we assume that the emitters operate independently of each other, each frequency band can be treated as a virtual sensor, each with its own sensing needs. The sensing needs of one sensor can conflict with other sensors, since more than one sensor can request the same resource at the same time. However, since we have only one receiver, only one of the sensors can be active at any specific time. Therefore, the requests for tuning to particular frequency bands must be scheduled appropriately, so that the receiver is sensing at the time when the illumination occurs.

Because of the large number of actual and potential emitters in an environment, it is important to minimize the time spent attempting to observe each emitter, while also maximizing the probability of detecting each illumination by an emitter. It is also 
important to keep frequency bands available for other uses, such as jamming. This results in additional pressure to reduce the time scheduled for the sensors to perform observations in each frequency band.

The problem considered in this paper is a great simplification (an abstraction) of real applications of this kind. However, since this is a non-preemptive scheduling problem, which is known to be NP-complete [2], a full and complete algorithmic solution to this problem does not exist. Consequently, algorithms that can push the limits in terms of the quality of solutions of this problem are sought. Moreover, this kind of scenario is of interest to other domains. For instance, a similar kind of problem is manifest in the managing of streaming media, which has similar characteristics.

A generally agreed upon model for sensor management (cf. [1]) is to view it as a loop consisting of three major functions: generate options, prioritize options and schedule tasks. The first function generates requests for sensor tasks, the second assigns priorities to the tasks, and the third then assigns the time of execution to each task. In this paper we consider all three functions. Options are generated separately for each sensor (frequency band). Priorities for tasks are assigned dynamically; the Quality of Service metric defined in the paper is used for this purpose. Note that the term "priority" refers here to a metric that the scheduler uses in selecting tasks for execution and not to the priority of a given emitter to the mission. The scheduler then fuses the information from all the virtual sensors and makes a decision on which sensor to schedule. Since in the subject domain the solution to this sensor management problem is termed as "scheduling", in the rest of this paper we use this term to mean inclusively "option generation, prioritization and scheduling".

The environment that we deal with is highly dynamic. Emitters are rotating and the receiver is located on a moving platform. In the general case, emitters can move, and illumination patterns can vary over time. Furthermore, events can occur over a very large range of time scales. Individual radiation pulses may be as brief as a nanosecond, while illumination periodicities can be as long as several seconds. More details about the scenario used in this study are given in Section 2. Additionally, since the scheduler 
is decoupled from the task generation and prioritization functions, the scheduler may introduce delays in scheduling the sensors (cf. [3]).

To compensate for the dynamics of the environment and of the system, we build and use a resource manager based on the control theory metaphor of software development [4]; we call this manager Dynamic Scan Scheduler (DSS). We formalize the dynamics of this resource allocation problem in Section 5.2. In order to map the problem onto the control architecture, we must also have a well defined quality of service (QoS) metric that can be used both to specify the required level of service and to measure the actual level of service achieved. The QoS metric used in our study is discussed in Section 5.3.

The next step is to choose a suitable control architecture and controllers. Our methodology is based on a general architecture for self-controlling software [4] which is specialized to this particular resource management problem. In this case, we chose a two-level feedback control architecture. We discuss our rationale for this choice in Section 5.1.

Finally, we evaluate our solution and compare it with other approaches used to solve this particular problem. The results of our simulations and comparisons are presented in Section 6. One approach is to develop a schedule off-line in advance of a mission. This type of resource manager, called a fixed scan scheduler (FSS), is introduced in Section 3. An algorithm for constructing fixed scan schedules is presented, and some examples are given. This technique guarantees detection of each illumination for low loads, i.e., when the number of emitters in the environment is small. This technique does not provide graceful degradation when the load becomes high.

We also consider another technique, periodic dynamic scheduling, that relies on prior knowledge about the frequency of illumination of the emitters. This technique is introduced in Section 4. This technique is dynamic but not adaptive. We show that the control based scheduler outperforms both of the other approaches. Furthermore, our solution uses only about $10 \%$ of the observation time as the fixed scan schedule, and our solution does not depend as much on the prior knowledge about the emitters as the periodic dynamic scheduling technique. The main reason for the performance advantages 
of our solution is that neither of the other two schedulers can adapt to dynamic changes in the environment. In our scenario, from the point of view of the receiver, the emitters are only quasi-periodic (due to the motion of the platform) and thus the sensor manager must compensate for the phase shift in illumination due to the motion. We end the paper in Section 7 with our conclusions and an outline of future research directions.

Sensor management has been formulated as an optimization problem in [5]. Since then various attempts have been made both to refine the formulation and to solve this problem. One of the directions was to use information theory based performance metrics $[6,7]$. However, Musick and Malhotra [1] state that "decision theoretic methods do not directly support scheduling". Another way to approach this problem is to treat it as a Dynamic Programming problem (cf. [1]). However, as stated in [1], "dynamic programming does not begin to resolve the modeling complexities innate in Sensor Management". Moreover, in our scenario we cannot even assume that we know the state transition function and thus estimation of the future states is necessary. When combined with the requirement of non-preemption of scheduling, this formulation does not solve our problem (one of the reasons being the NP-completeness of non-preemptive scheduling mentioned above).

Control techniques have also been applied to sensor management in various ways, e.g., $[3,6]$, but mainly for scenarios where the goal was to manage sensors used for tracking moving objects. One of the distinguishing features of our scenario is that our system has discrete input (detection or non-detection). Although similarly as in [3] in our scenario we also have an architecture in which the scheduling function is separated from the option generation function, and thus we have a similar problem with the delays due to this architecture, we could not apply these results to our scenario mainly because these results are related to tracking, rather than detection.

Feedback and control based algorithms have also been used in the area of real-time systems, where there is a need for scheduling the processor for computational tasks (cf. $[8,9,10,11]$ ). This work, however, addresses only one of the problems of sensor management, i.e., the scheduling part. 


\section{Scenario and Notation}

In this section we describe the simulated scenario in more detail, especially the simplifying assumptions we made, and introduce some notation used throughout the paper.

In Figure 1 we show a typical emitter illumination pattern. The radiation is produced in short pulses. These, in turn, form batches called illuminations. The pulses are shown here as square pulses, but the actual pulses are high frequency waveforms. The line over each illumination group is meant to represent the envelope of the illumination. It is not another signal.

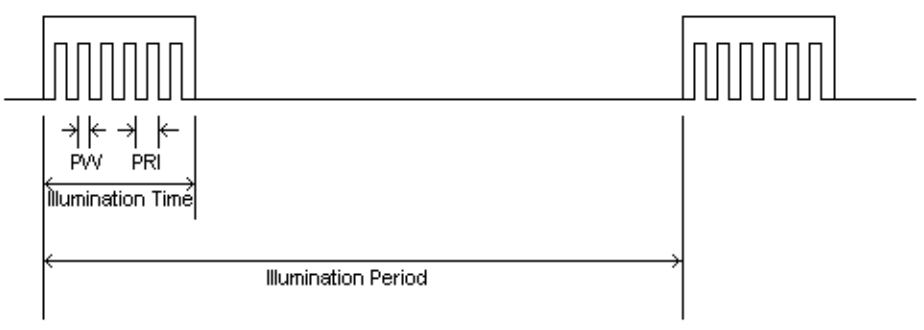

Figure 1: Signal

While the pulses are produced electronically, we assume that the illuminations are the result of the mechanical motion of the radar antenna. This has some important consequences. The time between pulses can be very short and can be very regular. On the other hand, the time between successive illuminations is typically much longer and need not be as regular. These assumptions are not valid for other types of emitter, for instance for phased array antennas, where any illumination pattern is possible. We do not consider this kind of emitters in our scenario.

The most important emitter parameters are the following: $f_{e}$ - the frequency of the emitter's signal (waveform); $A_{e}$ - signal strength (power level) of the emitter; $T_{p r i}$ - Pulse Repetition Interval (PRI) of the emitter (time between successive pulses within a single illumination); $T_{p w}$ - pulse width $(\mathrm{PW})$ of the emitter; $T_{\text {eip }}$ - the illumination period (time between successive illuminations, determined by the rotation period of the radar); $B_{e}$ beam width of the emitter (angle scope within which the emitter is radiating at a given 
time, determines the angle within which a target can be detected); $T_{e i t}$ - illumination time (duration of a single illumination); $\tau_{e}$ - the minimum time required for a detection of the emitter against background noise.

We assume that the kinds of emitter (radar) that might be encountered during a mission are known in advance. In particular, we assume that the radar monitoring system has basic data on the frequencies used by the emitters and the times between successive illuminations when the emitters are operating. Obviously, we do not assume that the phases for particular radars are known.

Another simplifying assumption is that the monitoring system can recognize the type of radar by its pulse pattern. This is not necessarily true in more complex and realistic scenarios. Nevertheless, in our scenario we assume that when an emitter has been detected, an attempt is made to identify the kind of emitter from a database of potential emitters. If this is successful, then the entry in the database is transferred to a table called the Active Emitter Table (AET). The AET is the primary output of the sensor, and it can also be used for scheduling the sensor. If the emitter cannot be identified as a known kind of emitter, then the system creates a new AET entry as well as a new database entry. This usually requires that the emitter be detected more than once in order to obtain an estimate for the illumination period.

Although we do not assume that there is at most one radar operating in each frequency band, we do assume that radars operating in the same frequency band can be distinguished from one another. The fact that this sensor operates in the same frequency band as another sensor does not make too much of a difference to the monitoring system.

As we already said in Section 1, even though this scenario is a great oversimplification of any realistic scenario, due to the fact that this is a case of non-preemptive scheduling, which is known to be NP-complete [2], it is worthwhile searching for algorithms that perform better in specific conditions. In our case the distinguishing conditions are that the goal is to detect each illumination of multiple emitters and that the system is operating under relatively high overload. 


\subsection{Tasks and Schedules}

Requests for tuning the receiver to the frequency band of a given emitter are called tasks. Each task is represented by a Control Description Word (CDW). A CDW specifies when and for how long the receiver is to be tuned to a particular frequency band. Each CDW is a request for a single dwell of the receiver. Mathematically, a CDW is a triple:

$$
C D W=\left(t_{d s}, \tau_{d}, \omega\right)
$$

where $t_{d s}$ is the time when the dwell should begin and $\tau_{d}$ is the length of time that the receiver devotes to this dwell. A scan schedule (SS) is a sequence of CDWs, one per dwell:

$$
S S=\left\{C D W_{1}, C D W_{2}, \ldots, C D W_{n}\right\}
$$

A scan schedule is simply the sequence of commands given to a receiver. Within a scan schedule, one can define the notion of the revisit time, $\tau_{r v}$, the time from the beginning of the last dwell until the beginning of the next dwell on a particular frequency band. From the beginning time of the last dwell and the revisit time, one can compute the start time of the next dwell.

The most basic constraint that a schedule must satisfy is called the capacity constraint. This constraint simply states in mathematical form the fact that a receiver can only be tuned to one frequency band at a time. For the virtual sensors it means that only one sensor can be scheduled at a time. For a scan schedule for which the number of frequency bands is a fixed number $N$ and for which the dwell time and revisit time for each frequency band $i$ is fixed, the capacity constraint is:

$$
W=\sum_{i=1}^{N} \frac{\tau_{d}(i)}{\tau_{r v}(i)} \leq 1
$$

The quantity $W$ is called the workload. Another way to view the capacity constraint is to introduce the notion of the duty cycle; it is the fraction of the time allocated to this emitter. More precisely, it is the ratio of the dwell time to the revisit time for this emitter. The capacity constraint states that the total of all duty cycles can be no larger than 1 . 
The satisfaction of the capacity constraint is not sufficient to ensure that a scan schedule exists. This is because the revisit times of different frequency bands may cause scheduling conflicts: two or more frequency bands may request the receiver at the same time. For this reason scheduling is an important part of the sensor control.

\section{$3 \quad$ Fixed Scan Scheduling}

One of the techniques of monitoring all illuminations of radars is the Fixed Scan Scheduling technique. A fixed scan schedule consists of a fixed sequence of CDWs for the entire mission of the platform. In this sequence, the CDWs for a specific emitter differ only in the start time $t_{d s}$, i.e., all the dwell times $\tau_{d}$ and the revisit times $\tau_{r v}$ for that emitter are the same. The main idea behind fixed scan scheduling is that in order to guarantee detection of each illumination, the receiver dwells should be spaced apart by no more than the illumination time of the emitter. With multiple emitters, this requirement is difficult to satisfy since multiple emitters might require dwell at the same time.

There are several ways to construct an fixed scan schedule. A rate monotonic fixed scan scheduler $(\mathrm{RM})[12,13,14,15]$ is a general task scheduling technique that uses two pieces of data about tasks: task execution time and period. It is a priority-based scheduling technique which assigns the highest priority to the task with the shortest period. For sensors, the execution time is the dwell time and the period is the revisit time. If one assumes that the dwell time is equal to $K$ pulse repetition intervals (where $K$ is typically equal to 3 ), then the following equations describe the rate monotonic scan scheduler:

$$
\begin{gathered}
\tau_{d}=K \cdot T_{p r i} \\
\tau_{r v}=T_{e i t}-\tau_{d}
\end{gathered}
$$

This assumes that the emitters emit pulses in a uniform pattern. It would not apply to emitters that use a non-uniform pattern, such as radars that employ a stagger pattern 
in which a non-periodic pattern of pulses is emitted repeatedly.

Figure 2 is an example of a rate-monotonic schedule. This schedule includes four emitters (E1, E2, E3, E4). Their characteristics (dwell time, illumination time) are given by: E1 : $(0.5,4)$, E2 : $(0.3,6)$, E3 : $(0.8,8)$ and E4 : $(0.6,12)$, respectively. Since RM scheduling gives higher priority to tasks with shorter periods, the priorities for these four emitters are 4, 3, 2 and 1 , respectively.

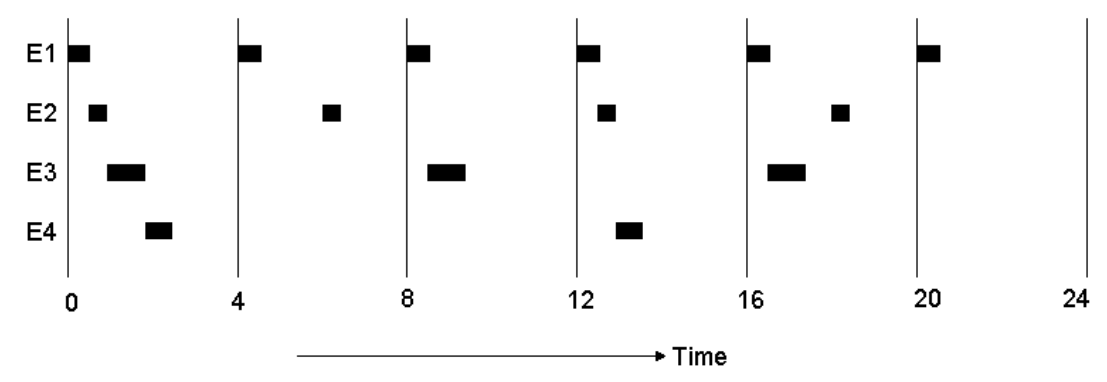

Figure 2: Example: A Fixed Scan Schedule

The computation of a rate-monotonic FSS starts by determining the least common multiple of the revisit times of the emitters. We call this the scheduling cycle. In the example of Figure 2, the revisit times are 4, 6, 8 and 12, so that the scheduling cycle is 24. In the next step, the CDW for the emitter having the highest priority is specified. This process is then repeated for the other emitters in order of priority until the scan schedule has been determined for the entire scheduling cycle. If the capacity constraint (Equation 3) is violated, then some revisit times must be increased using some rule, such as increasing the revisit time of lower priority emitters, until a scan schedule satisfies the capacity constraint and a schedule can be found. The schedule developed for one scheduling cycle is then repeated for all consecutive cycles.

In the absence of any new information (i.e., in the absence of any emitter detections), an FSS has reasonably good performance, especially if the scenario is the same as was anticipated during the selection of a given schedule. A FSS has the advantage that it is simple and thus easy to implement. This technique is also important because it is usually the initial schedule for any other kind of scan scheduling. 
However, FSS is based on prior knowledge about the environment, so it is not applicable in unknown environments or environments that change. In particular, since it does not have a feedback mechanism, it cannot adapt to changes in the environment. Consider, for example, a rate monotonic FSS and a simple scenario consisting of a single emitter and a single sensor having the following parameters:

$$
\begin{gathered}
T_{p r i}=2.0 \cdot 10^{-3} \mathrm{sec} \\
T_{p w}=1.5 \cdot 10^{-5} \mathrm{sec} \\
T_{e i p}=4.0 \mathrm{sec} \\
B_{e}=2^{o}
\end{gathered}
$$

From these equations one can calculate the illumination time to be:

$$
T_{\text {eit }}=4.0 \mathrm{sec} \cdot\left(2^{\circ} / 360^{\circ}\right)=0.02 \mathrm{sec}
$$

The dwell and revisit times for a rate monotonic fixed scan schedule will then be:

$$
\begin{gathered}
\tau_{d}=3 \cdot T_{p r i}=6.0 \cdot 10^{-3} \mathrm{sec} \\
\tau_{r v}=T_{\text {eit }}-\tau_{d}=0.0162 \mathrm{sec}
\end{gathered}
$$

In a rate-monotonic FSS for this scenario, there are $246 \mathrm{CDWs}$ for this emitter in each scheduling cycle. This corresponds to a duty cycle of

$$
d=\tau_{d} / \tau_{r v}=0.37
$$

for just this one emitter. The 246 receiver dwells corresponding to these CDWs will detect at most two illuminations of the emitter. Thus only two dwells will be effective; the other 244 will be wasted. When there are tens or even hundreds of emitters in the environment, this kind of scheduling will not be efficient. The goal is to reduce the number of CDWs required to detect each emitter, or, equivalently, to reduce the emitter duty cycles. Note, however, that we cannot lower the duty cycles by enlarging revisit times, since this might result in non-detections of emitter illuminations. 


\section{Introducing Dynamics to a Scheduler}

One way to lower the number of dwells is to synchronize the dwells with the illumination period of a given emitter, after first detection of that emitter. However, in the dynamic environment we still are risking missing illuminations due to dynamic effects (phase shifts). To compensate for the dynamics of the environment, a dynamic scan scheduler (DSS) is then used. Rather than being a fixed scheduling pattern, a dynamic scan scheduler is an algorithm that dynamically modifies the scan schedule in response to detection events.

There are several ways that one can introduce dynamics into a scan scheduler. One possibility is to use a pre-computed fixed scan schedule until first detection, and then recompute an it so that the newly detected emitter is only dwelled upon at the predicted illumination times as specified by the entry in the AET. After recomputation the CDWs for a detected emitter differ only in the start time $t_{d s}$, i.e., all the dwell times $\tau_{d}$ for the frequency band of this emitter are the same. We will call this technique periodic dynamic scan scheduling. The only difference between this technique and FSS is that detected emitters will have a much longer revisit time.

While periodic dynamic scan scheduling seems like it solves the problem of resource allocation in this case, it has some serious disadvantages. The main problem is that the illuminations of the emitters, as sensed by the receiver, will vary over time for a variety of reasons. The radar antenna, being a mechanical device, could speed up or slow down. However, a more significant effect is due to the motion of the aircraft. As the aircraft moves relative to the emitter, the apparent time between illuminations

changes. For instance, consider an emitter with a 6 sec illumination period (i.e., the radar antenna rotates by one full cycle in $6 \mathrm{sec}$ ). Now suppose that the aircraft is 60,000 feet away from the radar and is moving in a circle around the radar at $1000 \mathrm{feet} / \mathrm{sec}$, in a direction opposite to the rotation of the radar. In such a case while the radar rotates by 360 degrees, the aircraft moves by 6,000 feet, which is equivalent to about 6 degrees. Consequently, the apparent time between illuminations will be shorter by approximately 
0.1 sec. This might seem like an insignificant shift, but since the shift is much higher than the illumination time, this shift is actually quite important. In fact, the illumination time is about $20 \%$ of the 0.1 sec shift, which is more than sufficient to cause a detection failure.

To address the problem of dynamic changes in the environment, we propose a controlbased DSS.

\section{Mapping to a Control Architecture}

We now show how the sensor scheduling problem can be mapped to a control based architecture. We first review some background in the control theory metaphor of software development. A specific mapping to a control theory architecture is then proposed.

\subsection{Control-Based Architecture}

The problem addressed in this paper appears at first to be an excellent candidate for the standard control theory based paradigm. In this paradigm the problem is formulated using a model, an optimal control law is derived using control theory and the system is proved to be stable. Unfortunately, this approach is not possible for the problem addressed in this paper for various reasons. First, like nearly all nontrivial software systems, this system is far too complex for a complete mathematical model. Second, the system is a hybrid system (i.e., it includes both continuous and discrete components). Finally, the system is nonlinear. Consequently, although formulation of the problem with a mathematical model and derivation of an optimal control law would be highly desirable, it is not possible to use the standard control theory methodology in this case. Faced with such a situation, one is tempted to abandon control theory entirely, and this is the most common approach. There are many examples of software that incorporates feedback processing without any reference to control theory at all. The approach used in this paper is in between these two extremes. We call it the control theory metaphor of 
software development (see [4]).

The main reason for proposing the control theory metaphor was to address the problem of changes in software requirements. In the case of embedded software, changes in software requirements come from changes in the environment with which the software interacts. As we discussed in Section 3, fixed scan schedules do not perform well when the environment changes dynamically or the workload is heavy. This is due to the high duty cycle required for each potential emitter and the relatively low probability of detecting each emitter. The control theory metaphor seems natural for this kind of application.

The main idea behind the control theory metaphor is to treat the basic software functionality as a Plant, to treat changes in the environment as disturbances, and to use feedback from the environment to adjust system behavior. In order to compensate for disturbances, a Controller must be added. From the point of view of software requirements, the Controller is a redundancy that is added to the system. The controller is not part of the original software requirements. Other kinds of (redundant) modules need to be added to implement a control loop, as shown in Figure 3.

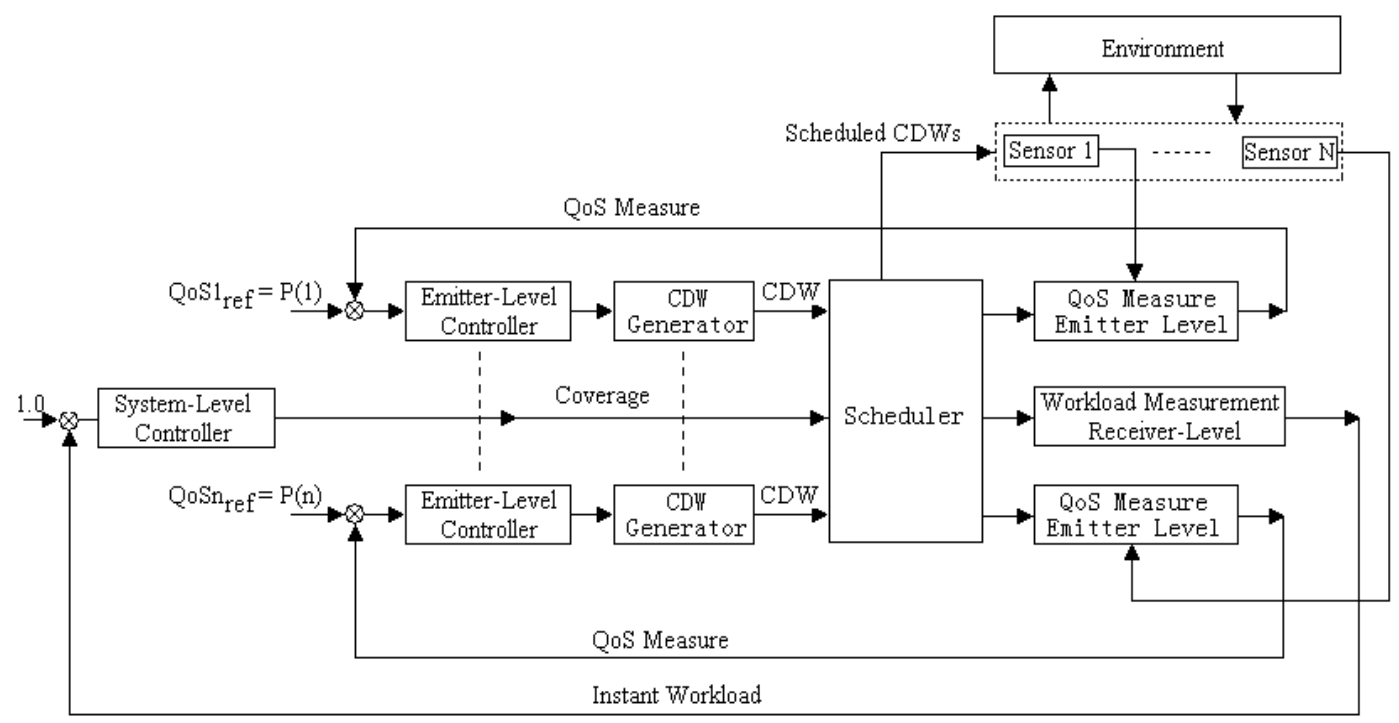

Figure 3: Control Based Sensor Management Architecture

In the control approach a system consists of two basic elements: a Plant (the system being controlled) and a Controller. To map a problem to a control architecture, it is 
necessary to identify a controlled output variable. In a control problem such a variable is always given explicitly. However, when the original problem statement does not include such a variable, an appropriate quantity needs to be constructed. The selection of this quantity is not a trivial task. In our approach we proposed to add a special Quality-ofService (QoS) module that assesses the Plant's output in terms of a Quality-of-Service measure. The selection of the QoS measure for our problem is discussed in Section 5.3.

\subsection{Plant}

Referring to Figure 3, the plant for this problem includes a receiver, a sensor management module and an environment. The receiver is viewed as a number of virtual sensors, one for each emitter. The sensor management module, similarly as in [1], consists of three functions: generate options, prioritize and schedule. In our mapping, the first two functions are included in the module called $C D W$ Generator (task generator). The scheduling function is obviously included in the Scheduler module. By environment, we mean the potential emitters. We don't have any control over the environment and in this sense this is not quite part of the plant. But, on the other hand, we partially model the emitters, and then the model is taken into consideration in further analysis. There are at least two reasons to discuss the model. First, we use the model in the selection of the controller parameters. Note, however, that it is not a typical derivation of a control law, like in a standard control-theory approach. Second, the model is used in the simulations used to evaluate the proposed approach.

\subsubsection{Emitters and Receiver}

The state of the platform and its receiver at a time $t$ is determined by the position of the platform and by the frequency band to which the receiver is tuned. The position of the platform is determined by its Cartesian coordinates $(x(t), y(t))$ over the terrain. The

altitude of the platform is not significant for this problem domain. The trajectory of the platform can be arbitrary but it is fixed in a particular mission so it is modeled as the 
input process of the dynamic system. The position as well as the velocity $\left(x^{\prime}(t), y^{\prime}(t)\right)$ of the platform can therefore be regarded as known.

The state of the receiver can be represented by a finite discrete set of frequency bands (sensors), along with one point representing the state in which the receiver is not tuned to any band. The current frequency band is written $\omega$, and the set of all such bands (including the "off" state) is written $\Omega$. The state space for the platform is therefore the product of the (continuous) two-dimensional plane and the set of frequency bands: $R=\mathbb{R}^{2} \times \Omega$.

We will assume that the emitters run independently. A state for a single emitter consists of the following three variables:

1. The direction $\phi$ in which the emitter is illuminating its environment. The variable $\phi$ is an angle, and therefore its set of values is isomorphic to the unit circle or to the half-open interval $[0,2 \pi)$.

2. The distance $r$ between the platform and the emitter.

3. The angle $\theta$ of the platform with respect to the emitter.

The state space for emitter $i$ will be denoted $E(i)$. The variables $r$ and $\theta$ represent the position of the platform with respect to the emitter in polar coordinates. It is sometimes convenient to use Cartesian coordinates, in which case they will be written $\left(x_{e}(i, t), y_{e}(i, t)\right)$.

Since the emitters were assumed to be independent, the overall state space for the emitters is the product of the state spaces of the individual emitters $\prod_{i} E(i)$. Combining this with the state space for the platform, the overall state space for the receiver and the emitters is:

$$
Q=R \times \prod_{i} E(i)=\mathbb{R}^{2} \times \Omega \times \prod_{i} E(i)
$$


The evolution function of the system has both continuous and discrete components. The continuous component evolution is determined by the following stochastic differential equation for emitter $i$ :

$$
\begin{gathered}
\frac{d}{d t} \phi(i, t)=2 \pi / T_{e i p}+w_{\phi}(i) \\
\frac{d}{d t} x_{e}(i, t)=x^{\prime}(t)+w_{x_{e}}(i) \\
\frac{d}{d t} y_{e}(i, t)=y^{\prime}(t)+w_{y_{e}}(i)
\end{gathered}
$$

In this system of equations, $w_{\phi}(i), w_{x_{e}}(i)$ and $w_{y_{e}}(i)$ are the noise affecting the respective derivative. For the radar rotation process the noise $w_{\phi}(i)$ is assumed to be Gaussian (with mean 0$)$. It is also assumed that $w_{\phi}(i)$ is independent of the other two noise variables.

The discrete component of the evolution of the receiver is characterized by discontinuous "jumps" from one state to another. The receiver evolution is determined by the scan schedule. Each CDW that is selected by the scheduler generates an event, which in turn, causes a discontinuous jump from one frequency band, $\omega_{i}$, to another, $\omega_{j}$, at the time of the event.

The last component of this dynamic system is the output function. This function determines what function of the variables of $Q$ are observed as the system evolves. In this case, the output function computes detections. Whenever the receiver satisfies the requirements for a detection, the function gives the data associated with the detection. In the following we define the detection function.

For each emitter $i$ there is an upper limit $r_{m}(i)$ on the distance at which it can be detected. When the platform is beyond the range of the emitter, then no detection is possible at any angle. The same would be the case if the emitter were not emitting either because it was not turned on or because the emitter was not deployed. For the purpose of this problem, it is not possible to distinguish an out of range emitter from one that is off.

The following are the requirements for detecting emitter $i$ : the emitter must be operating and within range, the emitter must be illuminating the receiver, and the emitter 
frequency must be in the current receiver frequency band. The requirements above must hold for a period of time at least equal to $\tau_{e}$. Putting the conditions above together we can formally define a detection as follows:

Definition 5.1 Consider a dwell commanded by the $C D W=\left(t_{d s}, \tau_{d}, \omega\right)$. The time $t_{d}$ is called the time of detection of emitter $i$, if there exists an interval $\left(t_{0}, t_{d}\right) \subset\left(t_{d s}, t_{d s}+\tau_{d}\right)$ such that for every $t \in\left(t_{0}, t_{d}\right)$

1. $r(i, t)<r_{m}(i)$,

2. $|\theta(i, t)-\phi(i, t)|<B_{e}(i)$,

3. $f_{e}(i) \in \omega(t)$, and

4. $t_{d}-t_{0} \geq \tau_{e}$.

The receiver actually measures the amplitude of the illumination continuously during the dwell time, and it determines that an illumination has occurred by simply comparing the measured signal with a noise threshold. For this reason the measurement noise will not be considered in the analysis of the model presented in this paper. A more elaborate model would, of course, consider the measurement noise in addition to the process noise.

When the receiver dwells on a specific emitter and does not detect that emitter, the output function returns $t_{l}(i)$, i.e., the time of last look at that emitter. The output function produces the set of detection times and look times for all emitters.

Definition 5.2 The output function $g: T \times \Omega \rightarrow T \times\{l, d\}$ is a function of time and frequency bands s.t., $g(t, i)=\left(t_{d}, d\right)$ if emitter $i$ was detected during last dwell and $\left(t_{l}, l\right)$, if emitter $i$ was not detected during last dwell.

For simplicity, we will say that the value of the function is either $t_{d}$ or $t_{l}$. Since the value of this function will not change between dwells, the set of detection times will be denoted as $T_{d}$ and the set of non-detections will be denoted as $T_{l}$. 
Note that this definition assumes that when more than one emitter is transmitting on the same frequency band, the receiver can distinguish them from each other. In other words, it can determine which of the potential emitters in a band is actually illuminating the receiver. Because emitters differ from one another in the detailed structure of the illumination (i.e., the pulse pattern), this is not an unreasonable assumption, although it might not always be correct. If such discrimination is not possible, then the output function must be redefined so that it only computes the frequency band that is being detected, not the specific set of emitters.

Since a detection can only occur during a dwell, one simplification of the output function is for the output function to produce a sequence of data structures, one for each CDW. This is, in fact, what many receivers produce. The output is a data structure called a pulse descriptor word (PDW) which contains detailed information about the illumination(s) that were detected during the period of time covered by the CDW.

\subsubsection{CDW Generator}

Another part of the plant is CDW Generator. We have one CDW generator for each emitter $i \in\{1, \ldots, N\}$. The goal of a CDW Generator is to generate requests for tuning to the frequency of the specific emitter (tasks), where each such task is represented by a CDW. Two parts of a CDW, $\tau_{d}$ and $\omega$, are fixed (cf. Equation 1). So the only value that may need to be recomputed is the time of the start of the next dwell, $t_{d s}(i, t+\Delta t)$, where $\Delta t$ is the period of the computation loop (in our simulations it was $10 \mu s e c$ ). It is re-computed at or after the beginning of the current dwell:

$$
t \geq t_{d s}(i, t)
$$

If the emitter illumination were perfectly periodic, if there were no phase shifts, if both the platform and the emitter were stationary, and if we knew the distance between the emitter and the platform then we would be able to predict exactly the time of the next dwell as the time of the current dwell $t_{d s}(t)$ plus the revisit time for that receiver 
$\tau_{r v}:$

$$
t_{d s}(i, t+\Delta t)=t_{d s}(t)+\tau_{r v}(i, t)
$$

In order to compensate for the phase shift, we need to adjust $\tau_{r v}$. This is achieved by using the input from the Emitter-Level Controller, $\delta(i, t)$ (see Figure 3). An intuitive way to achieve this adjustment is to multiply the controller output by the illumination period of this specific emitter, i.e.,

$$
\tau_{r v}(i, t)=\delta(i, t) \cdot T_{e i p}(i)
$$

However, since we use a generic proportional controller, the result of this multiplication could take the revisit time out of the desired bounds. To avoid this effect, and to add some nonlinearity (explained later) we replace $\delta(i, t)$ in Equation 21 with $\bar{\delta}(i, t)^{2}$,

$$
\tau_{r v}(i, t)=\bar{\delta}(i, t)^{2} \cdot T_{\text {eip }}(i)
$$

where $\bar{\delta}(i, t)$ is normalized by the following function:

$$
\bar{\delta}(i, t)= \begin{cases}1, & \text { if } \delta(i, t)>1 \\ \sqrt{\frac{T_{e i t}(i)}{T_{e i p}(i)}}, & \text { if } \delta(i, t)<\sqrt{\frac{T_{e i t}(i)}{T_{e i p}(i)}} \\ \delta(i, t), & \text { otherwise }\end{cases}
$$

As we can see from this equation, in case the controller output is large, the value of the revisit time is equal to the illumination period, i.e., the receiver dwells on a given emitter only once per the illumination period. This kind of situation should occur only when there was a detection on the previous dwell. When the controller output is small, the receiver dwells on the emitter after one illumination time. This is then equivalent to having an FSS sensor manager. Moreover, the normalization function is nonlinear. This has the effect of generating more CDWs closer to the next expected illumination. An example of this effect on the sequences of CDWs for a control based DSS is shown in Figure 4 in Section 5.4.3, where we discuss design issues of such sensor managers. The figure also shows a pattern of CDWs generated by an FSS. Note that since the CDW Generator shifts starts of dwells, this module also introduces additional dynamics to the system. 


\subsubsection{Scheduler}

The scheduler is responsible for combining information about all emitters and then adjusting the workload and selecting CDWs to be executed by the receiver. The CDWs generated by the CDW Generators are fed into the scheduler's queue. However, since the CDWs are generated by a number of independent generators (one per emitter), there may be too many CDWs for this receiver to execute. The limit on the capacity of the receiver is defined by the instant workload; it is violated (i.e, the scheduler is overloaded [16]) if the value of the workload is higher than one (see Equation 3). In other words, the set of tasks violates the schedulability constraint [17]. The instant workload is defined as the sum of the duty cycles of the CDWs in the queue:

$$
W(t)=\sum_{i \in\{C D W\}} \frac{\tau_{d}(i)}{\tau_{r v}(i, t)}
$$

where $\{C D W\}$ denotes the set of CDWs in the queue. The scheduler adjusts the workload by adjusting the revisit time for each CDW in the queue as follows:

$$
\tau_{r v}^{\prime}(i, t)=\frac{\tau_{r v}(i, t)}{c(t)}
$$

where $c(t)$ is the coverage parameter computed by the system-level controller. When the scheduler receives a larger value of $c(t)$ from the system-level controller, the revisit times of all emitters get smaller (Eq. 24), which results in larger duty cycles, and consequently in a larger instant workload (Eq. 23).

The scheduler is invoked at schedule time, $t_{s}$. The schedule time is defined as the completion time of the current dwell, i.e., $t_{d s}(t-\Delta t)+\tau_{d}(t-\Delta t)$ or the beginning of the next dwell in the queue. We use a priority-based scheduler, where priorities are based upon the Quality of Service metric computed dynamically. A scheduling decision $\omega\left(t_{s}\right)$ is made based upon the current value of the QoS (which will be defined in Section 5.3), where a specific frequency band $j$ is chosen if the QoS for it is the largest, i.e.,

$$
\begin{gathered}
\omega\left(t_{s}\right)=j, \\
Q o S\left(j, t_{s}\right)=\max _{i} Q o S\left(i, t_{s}\right)
\end{gathered}
$$




\subsection{The Quality of Service Metric}

The QoS is a metric that determines the level of quality exhibited by the system at any point in time. It can be used to specify the required level of service of the system and to compare the performance of different algorithms. As noted in Section 5.2 above, the purpose of the system is to keep the QoS at a given (low) level. In this section we define this metric. First, we introduce the emitter-level QoS $(i, t)$ metric (for an emitter $i$ at time $t$ ) and then combine all the emitter-level metrics to obtain a system-level metric $Q o S(t)$.

The goal of this sensor system is to detect each illumination of each radar. The success of this operation depends on the knowledge of one of the state variables of each emitter, the beam direction of the emitter. Therefore, we want the QoS function to measure the accuracy of the estimates of this state variable. Since the dynamic system for each emitter is a linear stochastic dynamic system (cf. Section 5.2.1), the overall measure of accuracy is the (co)variance of the error estimates.

The rotation of the emitter, which is a mechanical system, is subject to mechanical noise. The noise term is written $w_{\phi}(i)$ in Equation 15. We assume that $w_{\phi}(i)$ is independent of $w_{\phi}(j)$ for $j \neq i$. We also assume that the variance of $w_{\phi}(i)$ is time-independent. It follows that the variance $\sigma_{b}^{2}(i, t)$ of the (error of the) beam direction estimate increases linearly with time, if the emitter is not being observed.

When the receiver is tuned to a frequency band containing the frequency of emitter $i$, then the emitter is being measured. The measurement is extended over a period of time (the dwell time). As a result of the nonlinearity of the measurement process, the gain due to the measurement varies with the beam direction as well as the distance from the emitter. For example, when the emitter is illuminating the platform, the gain will be much higher than when the emitter is not.

One can summarize these observations as follows:

1. When emitter $i$ is not being observed, its emitter-level QoS increases linearly with 
time.

2. When emitter $i$ is being observed, its emitter-level QoS decreases with time, but the decrease depends on the result of the measurement.

A similar model for sensor management was introduced in [18], but in that model the measurement process was assumed to be linear. As a result, the reduction in the variance was a linear function of time during a measurement.

As discussed in Section 5.2.1, we assume that the receiver measurement is processed to produce a discrete two-valued response (detection or non-detection). This simplifies the effect of a measurement on $Q o S(i, t)$ to just two cases: a large gain when the receiver detects an illumination and a small gain when the receiver does not detect an illumination.

To obtain an explicit formula for the emitter-level QoS metric, we make some assumptions and approximations. We first assume that the beam direction noise variance is inversely proportional to the rotation period. In other words, we presume that the error accumulates at the same rate per rotation for every emitter. Therefore, if one is not observing the emitter, its emitter-level QoS increases at the rate $\frac{P(i)}{T_{e i p}(i)}$, where $P(i)$ represents the rate of error increase; we made an assumption that this rate is equal to the priority of the emitter $i$.

We next assume that when the emitter is detected, its beam direction variance drops to 0 . This is only approximately correct, since a detection only determines the beam direction within an angular segment determined by the beam width.

The last case to consider is an observation of the emitter that does not detect it. In this case one has eliminated the possibility that the beam direction is in the interval covered by the beam width. This assumes that the dwell time is the minimum necessary for a detection. A longer dwell would reduce the variance by more than the beam width. Under these assumptions, an observation reduces the beam direction variance by an amount approximately proportional to the ratio of the beam width to the rotation period. For simplicity we assume the same constant of proportionality $P(i)$ as above. 
Under these assumptions, the net effect of such an observation on the emitter-level QoS is to reduce it by $P(i) \cdot \frac{T_{e i t}(i)}{T_{e i p}(i)}$.

We now summarize this analysis with the following formula for the emitter-level QoS metric:

$$
Q_{o S}(i, t)=\frac{P(i)}{T_{e i p}(i)} \cdot\left(t-t_{d}(i)\right)+d\left(i, t_{l}\right)
$$

where

$$
d\left(i, t_{l}\right)=\left\{\begin{aligned}
P(i) & : \quad t_{l}=\text { StartTime } \\
0 & : \quad t_{l} \in T_{d}(i) \backslash \text { StartTime } \\
d\left(i, t_{l}^{-}\right)-P(i) \cdot \frac{T_{e i t}(i)}{T_{e i p}(i)} & : \quad t_{l} \in T_{l}(i) \backslash T_{d}(i)
\end{aligned}\right.
$$

In this formula, $d\left(i, t_{l}^{-}\right)$is the "previous" time (at which the value of the QoS was computed $), t_{d}(i)$ denotes the time of last detection of emitter $i$, and $P(i)$ denotes the weight associated with this emitter. $P(i)$ is proportional to the importance of the emitter to a given mission; it may also be thought of as priority of the emitter. In addition, $T_{d}(i)$ denotes the set of detection times for this emitter at the start time, and $T_{l}(i)$ denotes the set of observation times for this emitter. We consider the observation time to be the time at the end of the dwell, so $T_{d}(i)$ is a subset of $T_{l}(i)$. Note that the function $d\left(i, t_{l}\right)$ is updated only at observation times.

The emitter-level QoS metrics can be combined to form the overall QoS metric (called the system-level QoS). The combination is usually either the maximum (or minimum) of the individual QoS metrics, or the (weighted) average of the individual QoS metrics.

Other researchers, cf. [19, 20, 6, 7], used an entropy based metric derived from information theory. Sensor allocation is then formulated as a linear programming problem. While this approach has the advantage of being generic and is well based in the theory of information, the linear programming formulation does not address the dynamics of this scenario. This scenario is closer to a dynamic programming formulation [21], rather than linear programming.

The real-time systems community uses the term "QoS" in a somewhat different way. The QoS there means "the level of service", i.e., the percentage of CPU time that is 
assigned to a particular task (cf. [9, 22, 23]).

\subsection{Controllers}

As shown in Figure 3, the system includes $N+1$ controllers: $N$ Emitter-Level Controllers (one per emitter) and a System-Level Controller. Other architectures are known in the subject literature (cf. [24, 25]), however not for the kind of problem that is addressed in this paper, i.e., monitoring illuminations of multiple radars. Consequently, we had to propose an architecture that fits the goals of our problem. One of the constraints here is that the controllers must be simple in order to allow for fast scheduling of sensors in real time.

\subsubsection{Emitter-Level Controllers}

The goal of the Emitter-Level Controllers is to compensate for the phase shift due to platform movement and scheduling delays. In the system described in this paper we simply used proportional controllers, with the proportional parameter denoted as $K(i)$. As shown in Figure 3, the control output, denoted as $\delta(i, t)$, is the input to the CDW Generator module.

The input to this controller is the difference between the reference input and the QoS computed by the QoS Measure Emitter Level module. Since the QoS represents uncertainty about illumination times, the reference input for the Emitter-Level Controller sets an expected level of uncertainty. In our simulations we set the reference input to the value of priority of a given emitter, $P(i) \in[0,1]$. This means that the input to this controller is:

$$
\epsilon=P(i)-Q o S(i)
$$

A different policy could be used to set the values of the reference signals. The advantage of the priority-based approach is that this results in more CDWs generated for the higherpriority emitters and thus illuminations of these emitters are less likely to be missed by 
the receiver.

The impact of the Emitter-Level Controllers on the probability of detection of illuminations can be explained as follows. First, if a given receiver dwell results in a non-detection, the QoS for this receiver gets larger (Eq. 27), meaning that the uncertainty about this emitter gets higher. This results in a smaller value of the controller input $\epsilon$ (Eq. 29), and consequently in a smaller value of the Emitter-Level Controller output $\delta(i, t)$ (since the controller output is proportional to $\epsilon$ ). In the CDW Generator, this makes the revisit time $\tau_{r v}(i, t)$ shorter (Eq. 21), i.e., more CDWs are generated for this emitter, which in turn means that the scheduler assigns the receiver to this emitter's band more frequently, hence increasing the probability of detection of an illumination.

\subsubsection{System-Level Controller}

The goal of the System-Level Controller is to compensate for changes in the demand for the workload (overload) of the receiver. In other words, the goal of this controller is to ensure that the constraint of schedulability [17] is satisfied when the scheduler is overloaded (similarly as in [16]). The output of this controller, $c(t) \in[0,1]$, is the input to the scheduler, as described in Section 5.2.3.

The input to the System-Level Controller is the difference between the reference signal and the system-level QoS. The system-level QoS is the overall instant workload defined by Equation 23. The reference input for the System-Level Controller is set to 1 . It is the maximal load for schedulability, or the capacity of the receiver.

We used a PID controller for system-level control. The proportional, integral and differential parameters for the PID controller are denoted as $K_{p}, K_{i}$ and $K_{d}$, respectively. The settings for these parameters are discussed in Section 5.4.4.

The impact of this controller on this sensor management system is as follows. When the System-Level QoS gets larger than 1, the System-Level Controller's input gets smaller, which in turn causes an increase of all the revisit times, and consequently in a smaller workload. The P component of this controller will increment the controller output pro- 
portionally to $K_{p}$, the I component will take into account previous values of the difference between the reference signal and the System-Level QoS, and the D component will add the increment of the control output proportional to the change in that difference.

The idea of controlling the demand for a scheduler is also known in the real-time scheduling community $[26,27,28]$.

\subsubsection{Emitter-Level Controllers: Design Issues}

The goal of the analysis in this section is to provide guidelines for designing EmitterLevel Controllers, i.e., selecting the controller parameter $K_{p}$. First we focus on the FSS. As we discussed in Section 3, the FSS dwells follow a fixed dwell pattern. The pattern is designed in such a way that the dwells are very frequent, only one illumination time apart. Figure 4 shows examples of dwell patterns for an FSS (middle) and for a DSS (bottom). Each vertical line represents a dwell. The top signal shows an example of two consecutive illuminations of an emitter. We can see from this Figure that only one or two of the CDWs result in a detection.

The DSS pattern is generated according to the model of the CDW Generator (Eqs. 21 and 22) and by the Emitter-Level Controller. This pattern shows dwells generated by the CDW Generator after the first detection of an illumination.

The goal is to select such a parameter $K_{p}$ that guarantees detection of each illumination in spite of the phase shift due to the motion of the platform. This can be achieved when the revisit time in the vicinity of the expected illumination is small, i.e.,

$$
\tau_{r v}(i, t) \leq T_{e i t}(i)-\tau_{d}(i)
$$

To formalize this we need to introduce a variable $\phi(i)$ (see Figure 4) representing the phase shift and a variable $\bar{t}(i)$ representing time of start of dwell after which the condition (30) must be satisfied. To achieve this, $\bar{t}$ must be before the start of the next illumination (see Figure 4). This is captured by the following condition:

$$
\bar{t}(i)-t_{d}(i)<T_{e i p}(i)-\phi(i)-\tau_{d}(i)
$$


Putting this all together, a consecutive detection of the illumination (after a detection of the previous illumination) is guaranteed if

$$
\forall_{t>\bar{t}(i)} \bullet \tau_{r v}(i, t)<T_{e i t}(i)-\tau_{d}(i)
$$

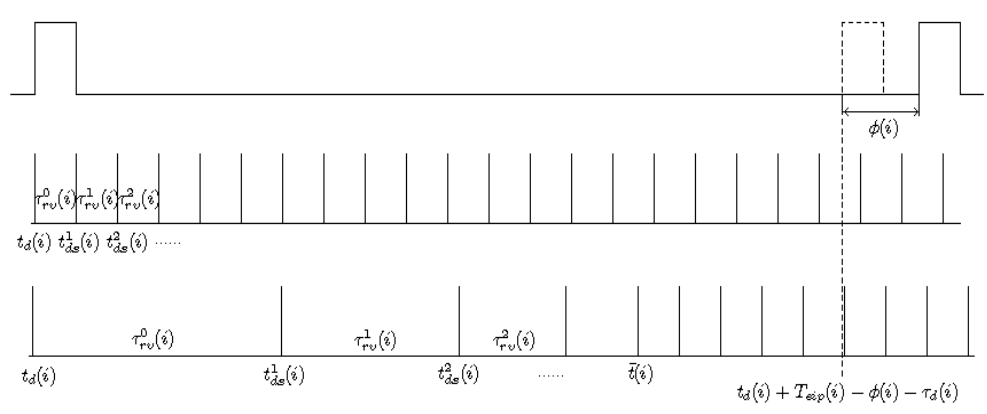

Figure 4: Example: Phase Shift and a DSS Dwell Pattern

To get an understanding of the role of the controller parameter $K_{p}(i)$ we show the following estimates. First we represent Equation 31 as:

$$
\frac{\bar{t}(i)-t_{d}(i)}{T_{\text {eip }}(i)}<1-\frac{\phi(i)}{T_{\text {eip }}(i)}-\frac{\tau_{d}(i)}{T_{\text {eip }}(i)}
$$

Assuming that $\frac{\phi(i)}{T_{e i p}(i)}$ is 0.02 (i.e., the phase shift is $2 \%$ of the illumination period, like in the example of Section 4) and that $\frac{\tau_{d}(i)}{T_{e i p}(i)}$ is negligible, we get the condition:

$$
\frac{\bar{t}(i)-t_{d}(i)}{T_{\text {eip }}(i)}<0.98
$$

In Table 1 we show how the choice of $K_{p}(i)$ affects the number of CDWs per one illumination period. We also show the value of the left hand side of Equation 34 to indicate that the condition defined by this Equation is satisfied.

If we knew the distance to a given emitter, provided that we know the illumination period of the emitter and the speed of the platform motion, we should be able to pick an appropriate $K(i)$, which guarantees detection while not overloading the receiver with too many requests for dwells (number of CDWs). However, if this kind of knowledge is not available, we need to select a more conservative approach. For an emitter with a long illumination period we can choose a controller with a large $K(i)$ resulting in fewer CDWs. For a system with a shorter illumination period, we can choose a controller with a small $K(i)$, which can generate more CDWs but will give a higher probability of detection. 


\begin{tabular}{|c|c|c|}
\hline$K(i)$ & $\frac{\bar{t}(i)-t_{d}(i)}{T_{e i p}(i)}$ & Number of CDWs \\
\hline 0.08 & 0.1191 & 198 \\
0.4 & 0.8268 & 63 \\
0.6 & 0.8851 & 42 \\
0.8 & 0.9125 & 31 \\
0.98 & 0.9604 & 9 \\
\hline
\end{tabular}

Table 1: Number of CDWs for Different $K_{p}(i)$

\subsubsection{System-Level Controller: Design Issues}

The discrete representation of the PID controller is given by the following equation

$$
C(Z)=K \cdot \frac{Z^{2}+a_{1} \cdot Z+a_{2}}{Z^{2}-Z}
$$

where

$$
\begin{gathered}
K=K_{p} \cdot\left(1+K_{i}+K_{d}\right) \\
a_{1}=-\frac{1+2 K_{d}}{1+K_{i}+K_{d}} \\
a_{2}=\frac{K_{d}}{1+K_{i}+K_{d}}
\end{gathered}
$$

The plant model can be approximated as:

$$
P(Z)=\frac{1}{z-1}
$$

The close-loop discrete transfer function of the whole control system is:

$$
H(Z)=\frac{K \cdot\left(Z^{2}+a_{1} \cdot Z+a_{2}\right)}{Z^{3}+Z^{2} \cdot(K-2)+Z \cdot\left(1+K \cdot a_{1}\right)+K \cdot a_{2}}
$$

Based upon this model, we can design a system with poles and zeros at $z 1=0.91, p 1=$ $0.885, p 2=0.565$, i.e., such that the poles and zeros are located within the unit circle, 
which results in a stable system. For instance, using Routh-Hurwitz method we can find the values of the PID parameters $K_{i}=0.5, K_{i}=0.1$ and $K_{d}=0.0$.

\section{Simulation Results}

We simulated a scenario in which a platform was moving along the straight line shown in Figure 5. The speed of the platform was 1000 miles/hour. It took the platform 230 sec to travel the path.

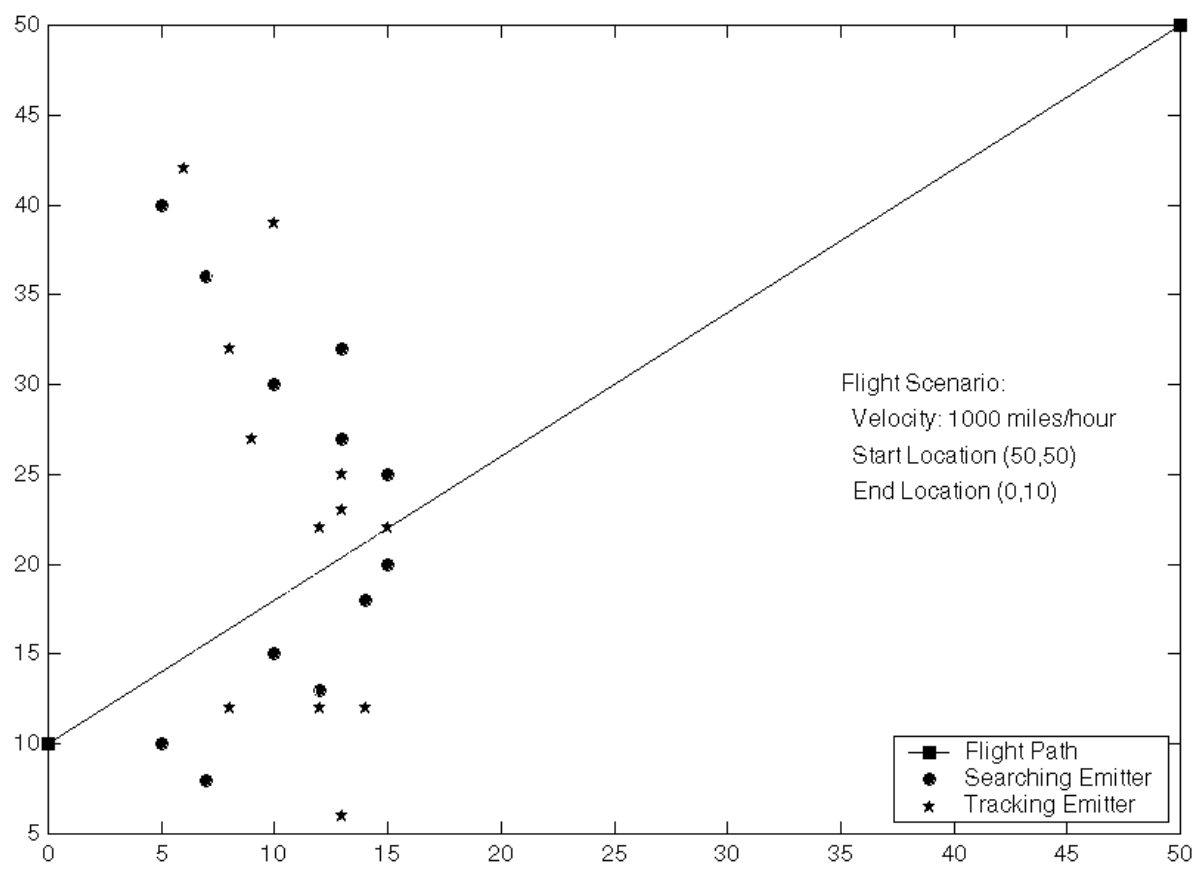

Figure 5: Simulation Scenario

There were twenty four emitters in the scenario. The characteristics of the emitters are shown in Table 2. We simulated both light and heavy load scenarios. Since for a light load the performance of a dynamic scan scheduler is roughly the same as for a FSS, we focused on heavy workloads.

The strength of the signal at the receiver was changing according to the following equation: 


\begin{tabular}{|c|c|c|c|c|c|c|}
\hline $\begin{array}{l}\text { Emitter } \\
\text { ID }\end{array}$ & $\begin{array}{c}f_{e} \\
G H z\end{array}$ & $\begin{array}{l}T_{p r i} \\
s e c\end{array}$ & $\begin{array}{l}T_{p w} \\
s e c\end{array}$ & $\begin{array}{l}T_{e i p} \\
s e c\end{array}$ & $\begin{array}{c}B_{e} \\
\text { degree }\end{array}$ & $\begin{array}{c}x, y \\
{[m i l e, m i l e]}\end{array}$ \\
\hline 1 & 2.25 & $2.0 \cdot 10^{-3}$ & $4.5 \cdot 10^{-5}$ & 10.0 & 4.0 & {$[5.00,10.0]$} \\
\hline 2 & 3.25 & $3.0 \cdot 10^{-3}$ & $3.5 \cdot 10^{-5}$ & 10.0 & 4.0 & {$[7.00,8.00]$} \\
\hline 3 & 5.25 & $4.0 \cdot 10^{-3}$ & $2.5 \cdot 10^{-5}$ & 10.0 & 4.0 & {$[5.00,40.0]$} \\
\hline 4 & 4.25 & $3.5 \cdot 10^{-3}$ & $1.3 \cdot 10^{-5}$ & 15.0 & 3.0 & {$[7.00,36.0]$} \\
\hline 5 & 6.25 & $2.5 \cdot 10^{-3}$ & $2.6 \cdot 10^{-5}$ & 15.0 & 3.0 & {$[10.0,30.0]$} \\
\hline 6 & 8.25 & $1.5 \cdot 10^{-3}$ & $3.9 \cdot 10^{-5}$ & 15.0 & 3.0 & {$[13.0,32.0]$} \\
\hline 7 & 7.75 & $2.5 \cdot 10^{-3}$ & $2.0 \cdot 10^{-5}$ & 18.0 & 3.5 & {$[10.0,15.0]$} \\
\hline 8 & 4.75 & $3.5 \cdot 10^{-3}$ & $3.0 \cdot 10^{-5}$ & 18.0 & 3.5 & {$[12.0,13.0]$} \\
\hline 9 & 5.75 & $4.5 \cdot 10^{-3}$ & $4.0 \cdot 10^{-5}$ & 18.0 & 3.5 & {$[15.0,25.0]$} \\
\hline 10 & 3.75 & $0.8 \cdot 10^{-3}$ & $4.0 \cdot 10^{-5}$ & 12.0 & 3.0 & {$[13.0,27.0]$} \\
\hline 11 & 2.75 & $1.6 \cdot 10^{-3}$ & $3.0 \cdot 10^{-5}$ & 12.0 & 3.0 & {$[15.0,20.0]$} \\
\hline 12 & 6.75 & $2.4 \cdot 10^{-3}$ & $2.0 \cdot 10^{-5}$ & 12.0 & 3.0 & {$[14.0,18.0]$} \\
\hline 13 & 11.25 & $2.0 \cdot 10^{-4}$ & $4.5 \cdot 10^{-6}$ & 5.0 & 2.0 & {$[8.00,12.0]$} \\
\hline 14 & 13.25 & $3.0 \cdot 10^{-4}$ & $3.5 \cdot 10^{-6}$ & 5.0 & 2.0 & {$[13.0,6.00]$} \\
\hline 15 & 15.25 & $4.0 \cdot 10^{-4}$ & $2.5 \cdot 10^{-6}$ & 5.0 & 2.0 & {$[10.0,39.0]$} \\
\hline 16 & 17.25 & $3.5 \cdot 10^{-4}$ & $1.3 \cdot 10^{-6}$ & 4.0 & 2.0 & {$[6.00,42.0]$} \\
\hline 17 & 16.25 & $2.5 \cdot 10^{-4}$ & $2.6 \cdot 10^{-6}$ & 4.0 & 2.0 & {$[9.00,27.0]$} \\
\hline 18 & 14.25 & $1.5 \cdot 10^{-4}$ & $3.9 \cdot 10^{-6}$ & 4.0 & 2.0 & {$[8.00,32.0]$} \\
\hline 19 & 12.25 & $2.5 \cdot 10^{-4}$ & $2.0 \cdot 10^{-6}$ & 6.00 & 2.5 & {$[12.0,12.0]$} \\
\hline 20 & 14.75 & $3.5 \cdot 10^{-4}$ & $3.0 \cdot 10^{-6}$ & 6.00 & 2.5 & {$[14.0,12.0]$} \\
\hline 21 & 16.75 & $4.5 \cdot 10^{-4}$ & $4.0 \cdot 10^{-6}$ & 6.00 & 2.5 & {$[13.0,25.0]$} \\
\hline 22 & 13.75 & $0.8 \cdot 10^{-4}$ & $4.0 \cdot 10^{-6}$ & 3.0 & 2.0 & {$[12.0,22.0]$} \\
\hline 23 & 15.75 & $1.6 \cdot 10^{-4}$ & $3.0 \cdot 10^{-6}$ & 3.0 & 2.0 & {$[15.0,22.0]$} \\
\hline 24 & 11.75 & $2.4 \cdot 10^{-4}$ & $2.0 \cdot 10^{-6}$ & 3.0 & 2.0 & {$[13.0,23.0]$} \\
\hline
\end{tabular}

Table 2: Characteristics of Simulated Emitters 


$$
A_{r}=\frac{A_{e} \cdot e^{-(\theta-\phi)^{2} / B_{e}{ }^{2}}}{r^{2}}
$$

where $A_{r}$ and $A_{e}$ are the signal strengths at the receiver and emitter, respectively, $\phi$ and $\theta$ are the angle of the beam line of the emitter and the angle between the receiver and the emitter, respectively, $B_{e}$ is the beam width, and $r$ is the distance between the receiver and the emitter. The illumination was detected if the strength $A_{r}$ of the signal at the emitter exceeded the noise threshold of the receiver.

In Figure 6 we show the distribution of the QoS for a FSS and for a DSS. The workload for this scenario was 2.7967. From this figure, we can see that the values of the QoS for the DSS are much lower than for the FSS. Since the QoS represents the uncertainty about the state of an emitter, the lower the QoS the better.

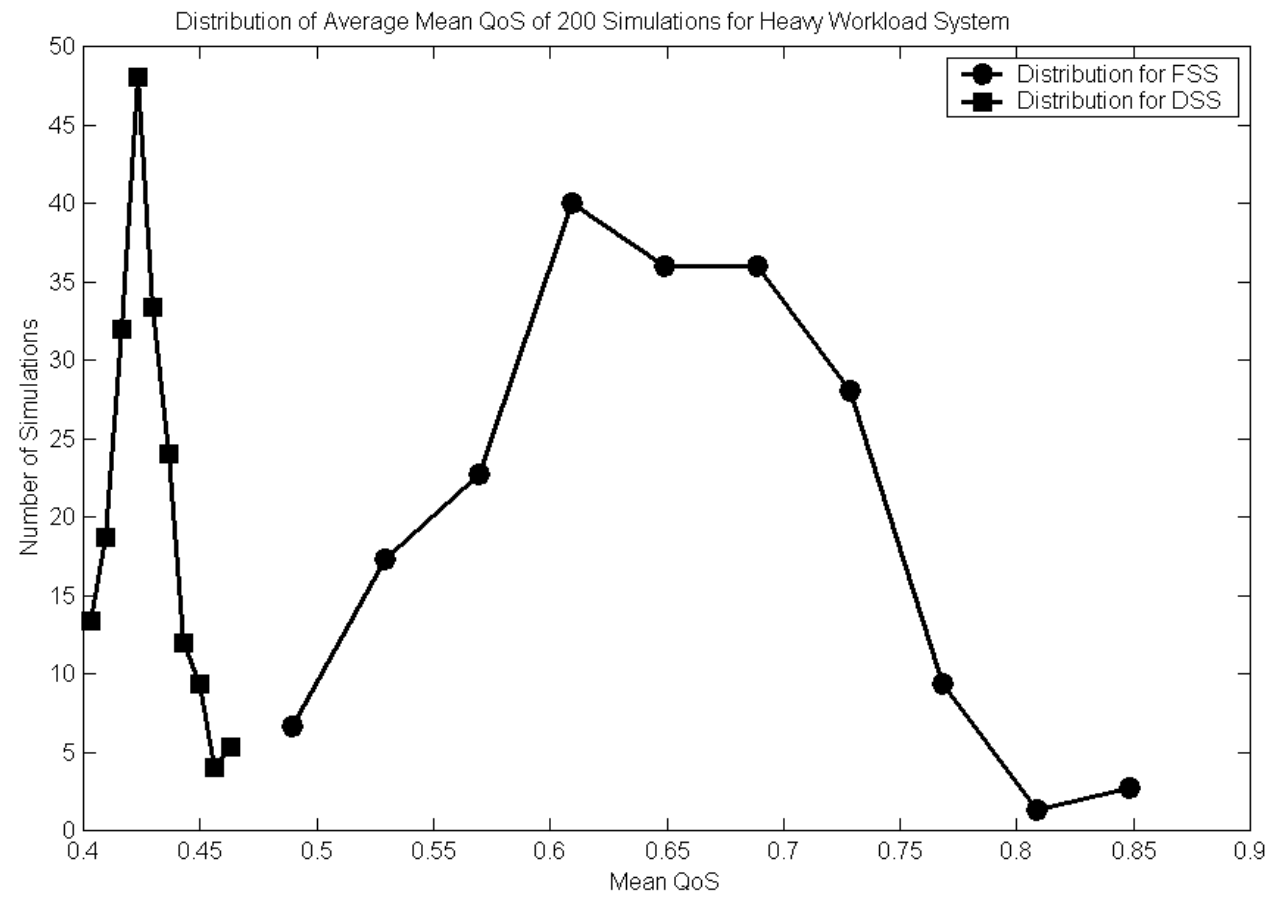

Figure 6: Distribution of Mean QoS; Workload $=2.7967$

In Figure 7 we show the distribution of the average maximal value of the QoS. From this figure we can see that the system responds to the controllers very well. The maximal value of the QoS does not cross the reference value of $P(i)$, which in this experiment was set to 1 for all emitters. The performance of the DSS is much better that of the FSS. The 
FSS's maximal QoS has a tendency to grow. The maximal QoS is an indication of the stability of the system. Roughly, the system is stable if the value of the QoS is bounded. Figure 7 shows that in the simulations the DSS showed a stable behavior.

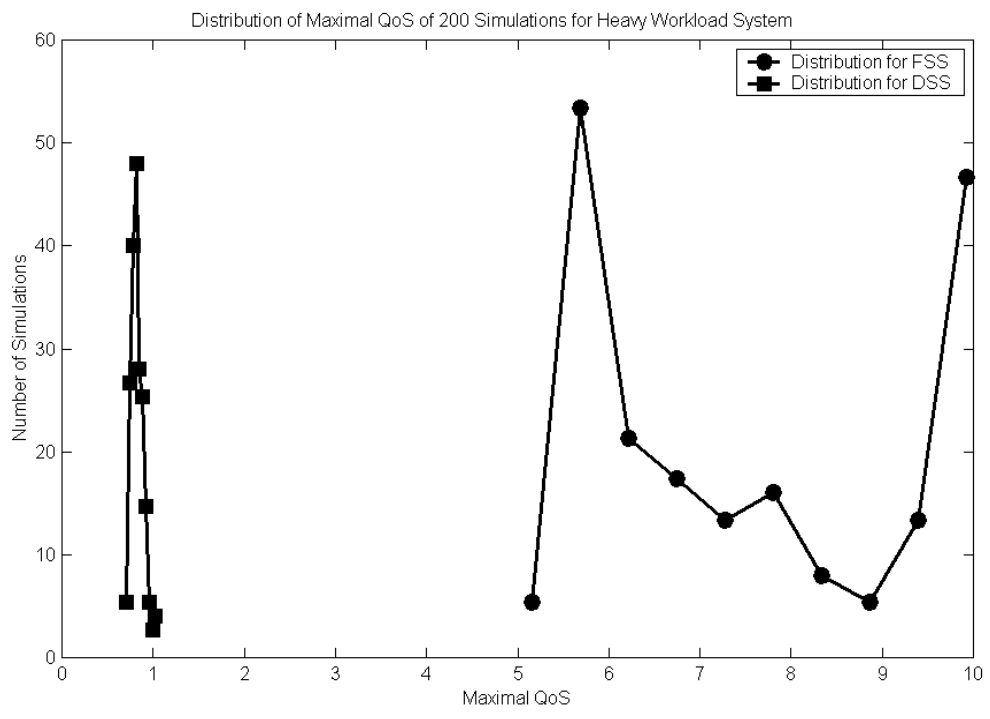

Figure 7: Distribution of Max QoS

\section{Conclusions and Future Research}

In this paper we present a new approach to receiver scheduling based upon the control theory metaphor of software development. We propose two metrics (the workload and the quality of service) used to provide feedback on the quality of performance of the scheduler and as controlled variables. These metrics are based on a simple and analytically tractable model of quality of service that is integrated into scheduling logic. We also propose to use a simple control logic for adjusting the parameters of a sensor scheduling policy with very limited model complexity, making it appropriate for fast real-time scheduling of sensors. We show that by an appropriate selection of the controller parameters the system exhibits a stable performance and discuss how to select such parameters.

The experimental results illustrate the value of the approach. From the results of the simulations it can be seen that the performance of the control based DSS is dramatically 
better than that of the FSS in both accuracy and the number of $C D W$ s especially when the system is overloaded. The QoS for the control based DSS is much lower and, at the same time, the number of $C D W \mathrm{~s}$ is reduced to only one tenth of the $C D W \mathrm{~s}$ in the FSS. This is understandable since our DSS does not assume that the illumination period is constant. Instead, it settles on a fixed period only through the application of its control law. This gives it the robustness that is especially important when the environment undergoes dynamic changes.

Although by using the control theory metaphor we achieved great improvement over the fixed scan scheduler, we still have room for optimization of this solution. For instance, we are implementing one more control loop to directly control the time spent for the computation of schedules. This is a very important issue in our application since the time scale of the scheduling is comparable to the time scale of computation. And thus the issue of complexity of computation needs to be addressed. Furthermore, we need to consider a more complex scenario in which there are multiple receivers as well as multiple emitters. This leads naturally to a distributed control architecture.

Another problem that needs to be addressed is the development of rules for mapping applications to the control theory based architectures. The mapping is currently being performed in an ad hoc manner based on experience. A more systematic approach would make it much easier for the control theory metaphor to be applied by the general software community. We made some advances in this direction in [18].

\section{Acknowledgments}

This research was partially supported by the Defense Advanced Research Projects Agency under contract F330602-99-C-0167. We are grateful to many people for helpful conversations and suggestions, most notably Kevin Passino, Mark MacBeth, Paul Zemany, Artan Simeqi and Fengming Zhang. Moreover, the we would like to express our gratitude to the anonymous reviewers whose constructive criticism helped us improve the clarity and the readability of this paper. 


\section{References}

[1] S. Musick and R. Malhotra. Chasing the elusive sensor manager. In Proceedings of the IEEE 1994 NAECON, Vol. 1, pages 606-613, 1994.

[2] M. R. Garey and D. S. Johnson. A Guide to the Theory of NP-Completeness. W. H. Preeman Company, 1979.

[3] L. Y. Pao and M. Kalandros. The effects of delayed sensor requests on sensor manager systems. In Proceedings of the AIAA Guidance, Navigation, and Control Conference, pages 1127-1135, 1998.

[4] M. M. Kokar, K. Baclawski, and Y. Eracar. Control theory-based foundations of self-controlling software. IEEE Intelligent Systems, pages 37-45, May/June 1999.

[5] J. Nash. Optimal allocation of tracking resources. In Proceedings of the 1977 Conference on Decision and Control, Vol. 1, pages 1177-1180, 1977.

[6] J. Llinas, K. J. Hintz, and G. O. Beale. Applications of automatic control theory to sensor scheduling. Technical Report NAWCADWAR-93002-50, Naval Warfare Center, Aircraft Division, Warminster, PA, 1992.

[7] W. Schmaedeke. Information based sensor management. In Proceedings of the SPIE Conference on Target Recognition and Data Fusion, Vol. 1955, pages 156-164, 1993.

[8] L. Palopoli, L. Abeni, F. Conticelli, M. D. Natale, and G. Buttazzo. Real-time control system analysis: An integrated approach. In IEEE Real-Time Systems Symposium, pages 131-140, Orlando, FL, 2000.

[9] C. Lu, J. A. Stankovic, T. F. Abdelzaher, G. Tao, S. H. Son, and M. Marley. Performance specifications and metrics for adaptive real-time systems. In IEEE Real-Time Systems Symposium, pages 13-23, Orlando, FL, 2000.

[10] D. C. Steere and et. al. A feedback-driven proportion allocator for real-rate scheduling. In Symposium on Operating Systems Design and Implementation, pages 145158, 1999. 
[11] C. Lu, J. A. Stankovic, G. Tao, and S. H. Son. Design and evaluation of a feedback control EDF scheduling algorithm. In IEEE Real-Time Systems Symposium, pages 56-67, Phoenix, AZ, 1999.

[12] J. W. S. Liu. Real-Time Systems. Prentice Hall, 2000.

[13] M. Klein, T. Ralya, B. Pollak, R. Obenza, and M. G. Harbour. A Practitioner's Handbook for Real-Time Analysis: Guide to Rate Monotonic Analysis for Real-Time Systems. Kluwer Academic Publishers, 1993.

[14] M. G. Harbour, M. H. Klien, and J. P. Lehoczky. Fixed priority scheduling of periodic tasks with varying execution priorities. Proceedings of IEEE Real-Time Systems Symposium, pages 116-128, 1991.

[15] J. P. Lehoczky, L. Sha, and Y. Ding. The rate monotonic scheduling algorithm - exact characterization and average case behavior. In IEEE Real-Time Systems Symposium, pages 166-171, 1989.

[16] P. Mejia-Alvarez, R. Melhem, and D. Mosse. An incremental approach to scheduling during overloads in real-time systems. In IEEE Real-Time Systems Symposium, pages 283-294, 2000.

[17] D. Seto, J. P. Lehoczky, L. Sha, and K. G. Shin. On task schedulability in real-time control systems. In IEEE Real-Time Systems Symposium, pages 13-21, 1996.

[18] M. M. Kokar, K. M. Passino, K. Baclawski, and J. E. Smith. Mapping an application to a control architecture: Specification of the problem. Lecture Notes in Computer Science, 1936:75-89, 2001.

[19] K. J. Hintz. A measure of the information gain attributable to cueing. IEEE Transactions on Systems, Man and Cybernetics, 21, No. 2:237-244, 1991.

[20] K. J. Hintz and E. S. McVey. Multi-process constrained estimation. IEEE Transactions on Systems, Man and Cybernetics, 21, No. 1:434-442, 1991. 
[21] D. A. Castanon. Approximate dynamic programming for sensor management. In Proceedings, IEEE Conference on Decision and Control, pages 1202-1207, 1997.

[22] R. Rajkumar, C. Lee, J. Lehoczky, and D. Siewiorek. Practical solutions for QoSbased resource allocation problems. In IEEE Real-Time Systems Symposium, pages 296-306, 1998.

[23] D. Hull, A. Shankar, K. Nahrstedt, and J. W. S. Liu. An end-to-end QoS model and management architecture. In IEEE Real-Time Applications Symposium, pages 176-189, 1997.

[24] C. Aurrecoechea, A Cambell, and L. Hauw. A survey of QoS architectures. In 4th IFIP International Conference on Quality of Service, pages 138-151, 2001.

[25] S. G. Bier and P. Rothman. Intelligent sensor management for beyond visual range air-to-air combat. In Proceedings of the IEEE 1988 NAECON, pages 264-269, 1988.

[26] G. Buttazzo, G. Lipari, and G. Abeni. Elastic task model for adaptive rate control. In IEEE Real-Time Systems Symposium, pages 286-295, 1998.

[27] D. Rosu, K. Schwan, and S. Yalamanchili. FARA - a framework for adaptive resource allocation in complex real-time systems. In IEEE Real-Time Technology and Applications Symposium, pages 79-84, 1998.

[28] D. Rosu, K. Schwan, S. Yalamanchili, and R. Jha. On adaptive resource allocation for complex real-time applications. In IEEE Real-Time Systems Symposium, pages 320-329, 1997. 